BNL - 66256

CAP-256-Muon-99

Pion Yield vs. Geometry of Target and $\sim 20$ T Pulse Solenoid for a Muon Collider Experiment

R.J. Weggel

Brookhaven National Laboratory

N.V. Mokhov

Fermilab

January 1999 


\title{
PION YIELD VS. GEOMETRY OF TARGET AND 20 T PULSE SOLENOID FOR A MUON COLLIDER EXPERIMENT
}

\author{
$\underline{\text { R. J. Weggel }}{ }^{\star}, \mathrm{BNL}^{\star}$, Upton, NY and N.V. Mokhov ${ }^{\star}$, FNAL*, Batavia, IL
}

\begin{abstract}
The Muon Collider Collaboration is proposing a pioncapture experiment that employs BNL's Alternating Gradient Synchrotron, a liquid metal target, and a pulse solenoid precooled by liquid nitrogen. This paper compares the yield with various target diameters, orientations and magnetic field protiles. To equalize costs, all magnets have the same mass, 12 metric tons. The magnet has two nested shells, energized sequentially. The outer set of coils, energized at 4 MVA, generates $\sim 1 / 3$ of the field in the target, most of the field downstream from it, and stores $\sim 21 \mathrm{MJ}$, from which to energize the inner coil.

The computer code MARS predicts that the meson yield 5 meters from the target can be $\sim 0.35$ per $16-\mathrm{GeV}$ proton. This is with a mercury target of $20 \mathrm{~mm}$ diameter and $0.3 \mathrm{~m}$ length, tilted $100 \mathrm{mr}$ from the field axis. The magnet field is $20 \mathrm{~T}$, averaged over the target, ramping downward as $(1+5 z)^{-1}$ over a $3 \mathrm{~m}$ length, while the bore increases inversely with the square root of the field.
\end{abstract}

\section{NEED TO MODEL PION CAPTURE}

To supply the particles for a muon collider [1] one needs three components. One is a source of nanosecond bunches of protons of multi-GeV energy. Another is a target in which the protons generate mesons ( $\sim 95 \%$ pions and $\sim 5 \%$ kaons), which decay into muons. The third component is a solenoid of large field and bore, to capture the mesons by bending their trajectories into helices spiralling around field lines. One seeks to maximize the yield of muons per unit proton energy, and to minimize the volume of their phase space, for the sake of high collider luminosity.

\section{TARGET \& PION-CAPTURE MAGNET}

The energy deposition from the proton beam is nearly instantaneous and may be of so high a density as to produce a violent shock wave in the target. A liquid jet is immune from permanent damage but, if electrically conducting, will decelerate and deform from Lorentz interactions with the field. To observe the behavior of the jet in the magnetic field, and thereby to assess its suitability as a target, is one goal of the pion-capture experiment.

\footnotetext{
"Email: weggel@bnl.gov

$\checkmark$ Supported by U.S. Dept. of Energy, contract \#DE-AC02-98CH10886

'Email: weggel@bnl.gov

*Work supported by the Universities Research Association, Inc., under contract DE-AC02-76CH00300 with the U.S. Department of Energy
}

To capture most of the forward-directed mesons generated in the target requires a large volume of intense field. Capture to a transverse momentum of $225 \mathrm{MeV} / \mathrm{c}$ needs 3 $\mathrm{T}-\mathrm{m}-\mathrm{e}$.g., $20 \mathrm{~T}$ in a bore of $0.15 \mathrm{~m}$. A continuous-field magnet of this performance is too expensive, either for the superconducting magnet or for the power supply. The constraint on power dictates a system pulsed from liquid nitrogen temperature, which improves the initial electrical conductivity by a factor of about seven. A crucial additional innovation is to employ two nested sets of coils, as in Fig. 1, energized sequentially. The modest power supply available to $\mathrm{BNL}, 16 \mathrm{kA}$ at $250 \mathrm{~V}$, energizes only the massive outer set of coils, which inductively stores $\sim 21$ MJ. Insertion of a $125 \mathrm{~m} \Omega$ resistor across its terminals then provides a voltage-initially $2 \mathrm{kV}$-to energize the inner coil quickly to high field.

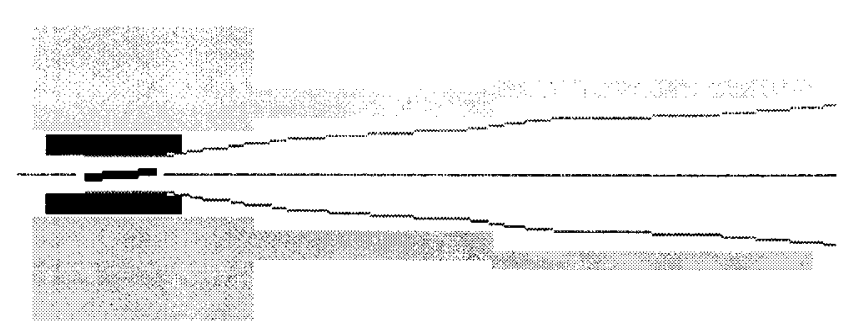

Figure 1: Pion-capture magnet, with average field $B_{0}$ in target, $-0.3<z<0$ meters. Magnet mass $=12$ metric tons. 4 $\mathrm{MW}, 21 \mathrm{MJ}$ outer set of coils energizes inner coil. Bore is $\sim 0.15 \mathrm{~m}$ near target, $\sim 0.6 \mathrm{~m}$ at mouth, $\mathrm{z}=\mathrm{L}$. For $0<\mathrm{z}<\mathrm{L}$, the field profile ramps downward as $B_{d} /(1+z / L)$. The cone is the envelope of captured mesons.

\subsection{Design of $\mathrm{LN}_{2}$-Precooled Pulse Magnet}

The prediction of magnet performance is complicated by the increase in resistance of each coil as it heats up during the pulse. Another complication is the inductive crosstalk between coils. This complexity precludes an analytical solution. Instead, one partitions the pulse into dozens of brief time intervals, in each of which one approximates the current, temperature and resistance of each coil as a power series of order 2 or more.

In a typical system, energizing the outer coil set takes about 20 seconds. Its peak field reaches $\sim 10$ teslas. Energizing the inner coil to its peak field of $\sim 13$ ' $\mathrm{T}$ ' takes only $\sim 0.3 \mathrm{~s}$. Meanwhile, the field from the outer set of coils has decayed to $\sim 7 \mathrm{~T}$, for a total of $\sim 20 \mathrm{~T}$. The total field is within $10 \%$ of its peak for $\sim 0.8 \mathrm{~s}$. When the inner coil has approximately doubled in resistance, insertion across its terminals of a resistor of $\sim 0.2 \Omega$ dumps its current, dissipaling most of its stored energy at room temperature and 
limiting its peak temperature rise to $\sim 40 \mathrm{~K}$. The total encrgy dissipated in the coils themselves is $-32 \mathrm{MJ}$ - enough to boil off 200 liters of liquid nitrogen. Figure 2 graphs these parameters for the $\mathrm{L}=3 \mathrm{~m}$ magnet of Fig. 1 .

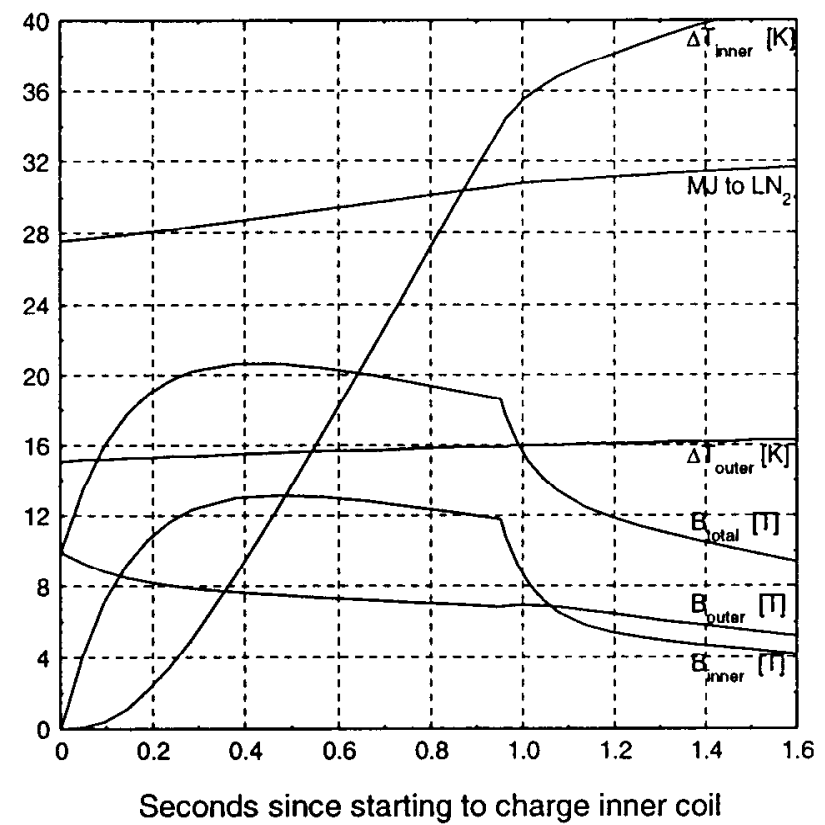

Figure 2: Field, temperature rise and cumulative heat dissipation vs. time in pulse magnet of Fig. 1 , in which a 4 MW outer set of coils energizes the inner coil. The fields are at $\mathrm{z}=-0.15 \mathrm{~m}$, halfway down the target.

\subsection{Peak Field vs. Field Profile of Magnet}

For the magnets of Fig. 1, with their fixed mass and peak power, the field within the target depends on the field profile further downstream-the more gradual the downramp (the greater the length $L$ ), the lower the peak field. Figure 3 shows that if the ramp length $L=2 \mathrm{~m}$, then $B_{b}$, the average field in the target, is $20.9 \mathrm{~T}$; if $\mathrm{L}=3 \mathrm{~m}, \mathrm{~B}_{0}$ is $20.0 \mathrm{~T}$; and if $L=5 \mathrm{~m}, \mathrm{~B}_{0}$ is only $17.3 \mathrm{~T}$.

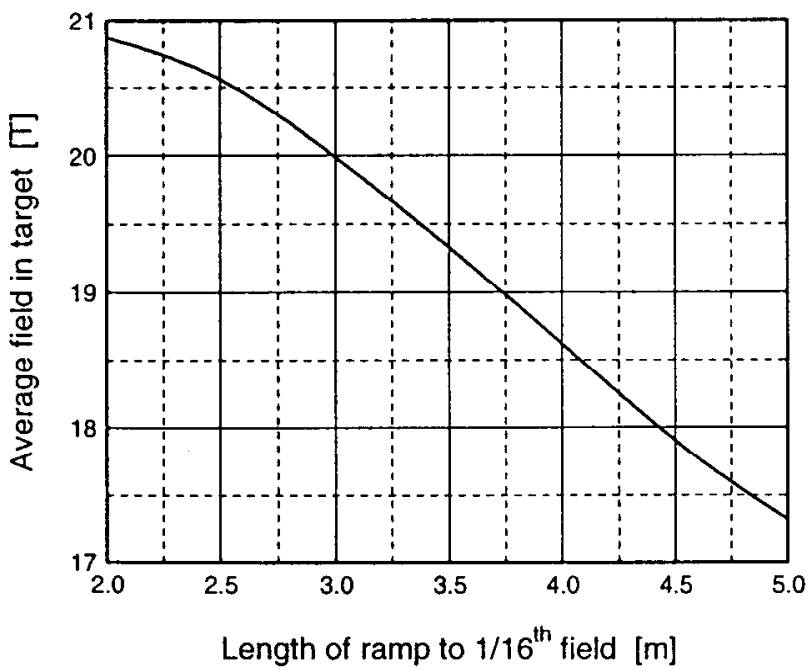

Figure 3: Field, averaged over the target, of the magnet of Fig. 1, vs. the length $L$ that defines the field profile, $\mathrm{B}(\mathrm{z})=\mathrm{B}_{4} /(1+15 \mathrm{z} / \mathrm{L})$, for $0<\mathrm{z}<\mathrm{L}$.

\section{PION YIELD AT $Z=L \& \&=5$ METERS}

The full Monte Carlo with the latest version of the computer program MARS(13)99 [2] predicts with unprecedented accuracy the production and trajectories of particles. The MARS simulation implements fully the target and magnetic field geometry as described above. Within the target the field is uniform, $B_{0}$; downstream, over a length $\mathrm{L}$, it decays sixteenfold as $\mathrm{B}(\mathrm{z})=\mathrm{B}_{0} /(1+k \mathrm{z}) \equiv$ $\mathrm{B}_{0} / \zeta$. For $\mathrm{z}>\mathrm{L}, \mathrm{B}=\mathrm{B}_{0} / 16$. The aperture radius $\mathrm{r}(\mathrm{z})=0.075$ $\zeta^{1 / 2}$. Maxwellian expansion, to $2^{\text {nd }}$ order, of the on-axis field gives the field components off axis as:

$$
\begin{gathered}
\mathrm{B}_{\mathrm{z}}(\mathrm{r}, \mathrm{z})=\left(\mathrm{B}_{0} / \zeta\right)\left[1-0.5(\mathrm{kr} / \zeta)^{2}\right] \\
\mathrm{B}_{\mathrm{r}}(\mathrm{r}, \mathrm{z})=0.5 \mathrm{~B}_{0}\left[\mathrm{kr} / \zeta^{2}\right]\left[1-0.75(\mathrm{kr} / \zeta)^{2}\right] .
\end{gathered}
$$

The program predicts the power dissipation in the target and in the first $50 \mathrm{~mm}$ radial depth of the solenoid windings, and predicts the residual dose rate in the target and solenoid. It also provides complete information on the particles at the solenoid mouth $\mathrm{z}=\mathrm{L}$, and at $\mathrm{z}=5$ meters. The target is tilted about its center, $z=-0.15 \mathrm{~m}$, by up to 150 milliradians. The particle momenta ranges are $0.05<\mathrm{p}_{\pi}<0.8 \mathrm{GeV} / \mathrm{c}$ and $0.1<\mathrm{p}_{\mu}<0.6 \mathrm{GeV} / \mathrm{c}$. Results are rather close to those with a simple $\mathrm{p}_{\mathrm{t}}<0.225 \mathrm{GeV} / \mathrm{c}$ cut.

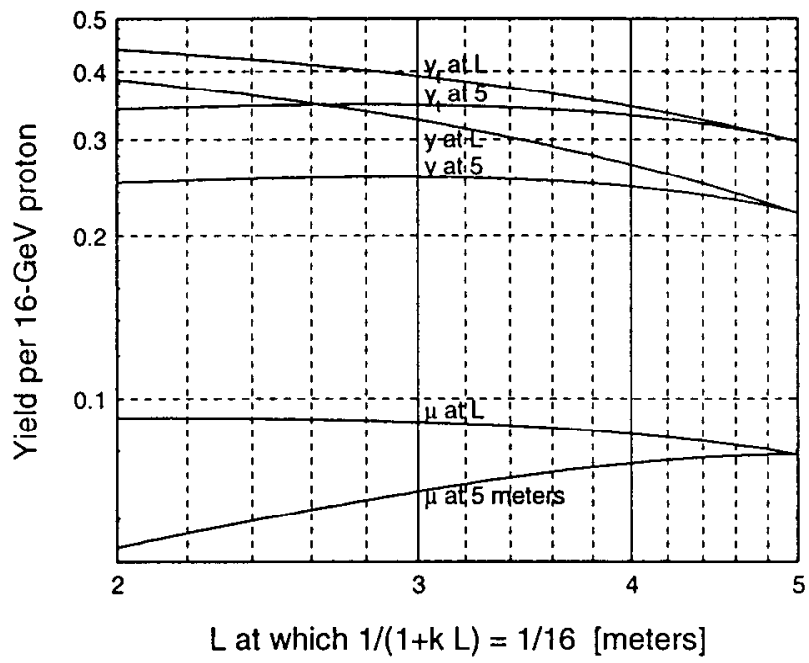

Figure 4: Meson yield per $16-\mathrm{GeV}$ proton. Beam: $\sigma_{x}=\sigma_{y}=$ $4 \mathrm{~mm}$. Target: $\mathrm{Hg}, 20 \mathrm{~mm}$ dia. by $0.3 \mathrm{~m}$ long, tilted $50 \mathrm{mr}$. $y \equiv \pi+K ; y_{t}=y+\mu$.

Pion momentum spectra at $\mathrm{z}=5 \mathrm{~m}$ (Fig. 5) are peaked at $p_{m}=200 \mathrm{MeV} / \mathrm{c}$. One can see about $20 \%$ more negative than positive pions when $\mathrm{p}_{\pi}<\mathrm{p}_{\mathrm{m}}$, and $20 \%$ fewer when $\mathrm{p}_{\pi}>\mathrm{p}_{\mathrm{m}}$. Most of the pions have rather low transverse momenta, with the distribution having two pronounced slopes: steep at $\mathrm{p}_{\mathrm{t}}<100 \mathrm{MeV} / \mathrm{c}$ and shallow at higher momenta (Fig. 6). Radial distribution of pion flux at $z=5 \mathrm{~m}$ is shown in Fig. 7. One sees that the entire system focuses generated pions rather well. It is true for produced muons and other particles, too. Good focusing properties of the 
proposed configuration are confirmed by a compact phase space-and emittance appropriately-as shown in Fig. 8 for pions at the exit from the matching solenoid.

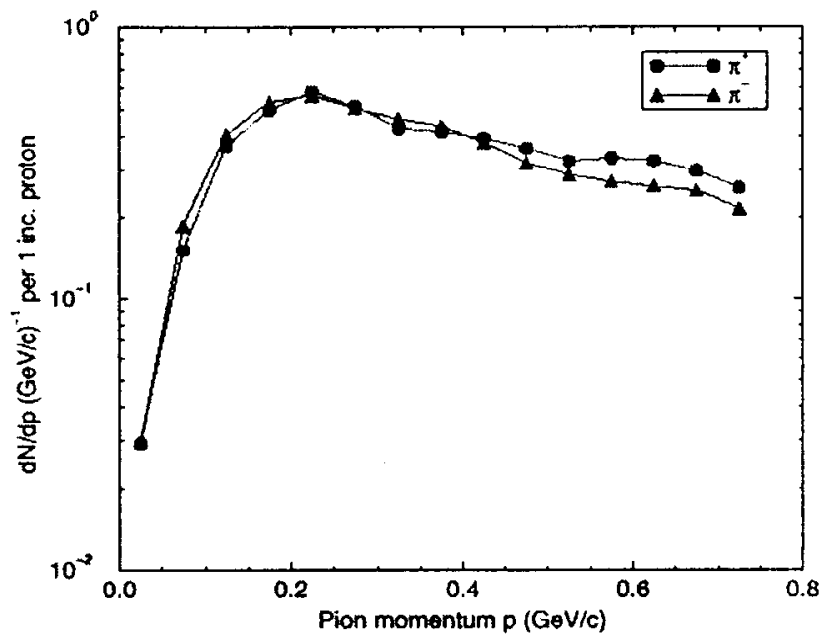

Figure 5: Pion momentum spectra at $\mathrm{z}=5 \mathrm{~m}$.

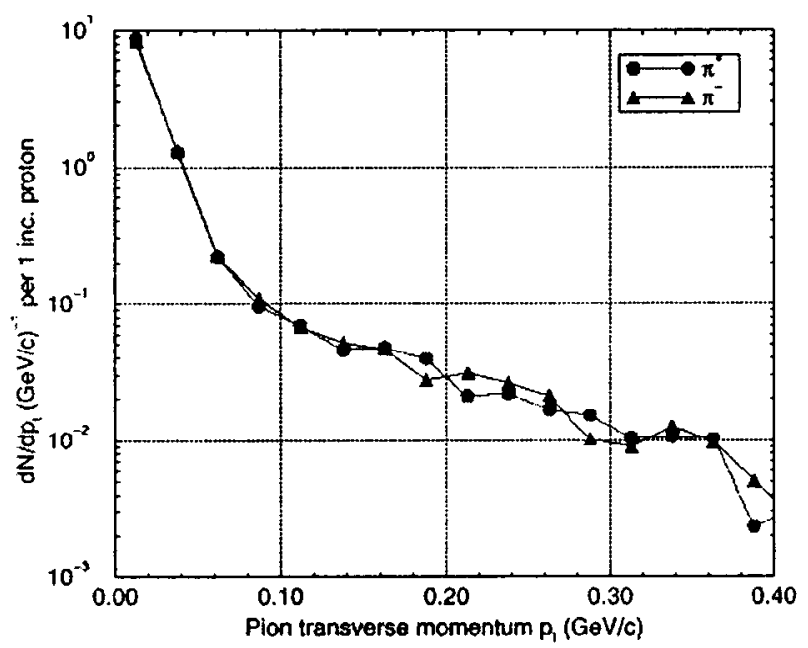

Figure 6: Pion transverse momentum distribution at $z=5 \mathrm{~m}$

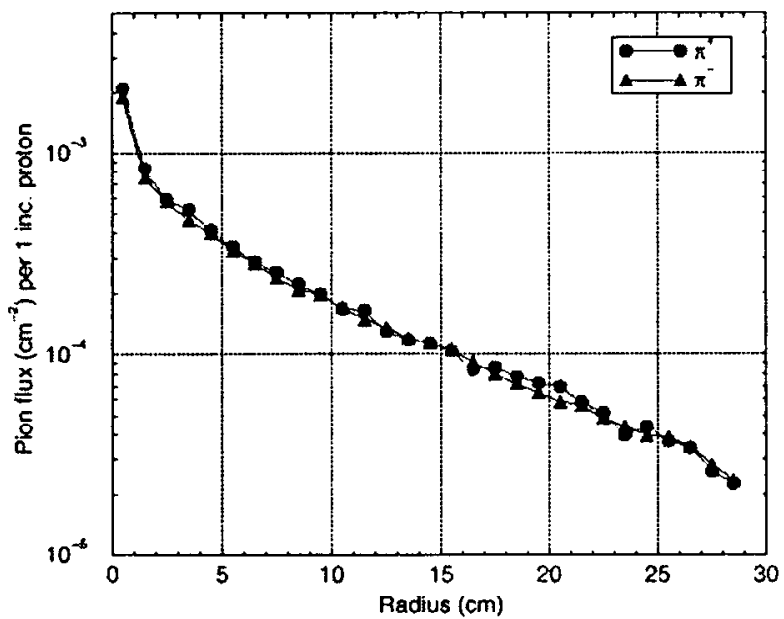

Figure 7: Lateral distribution of pion flux at $\mathrm{z}=5 \mathrm{~m}$.

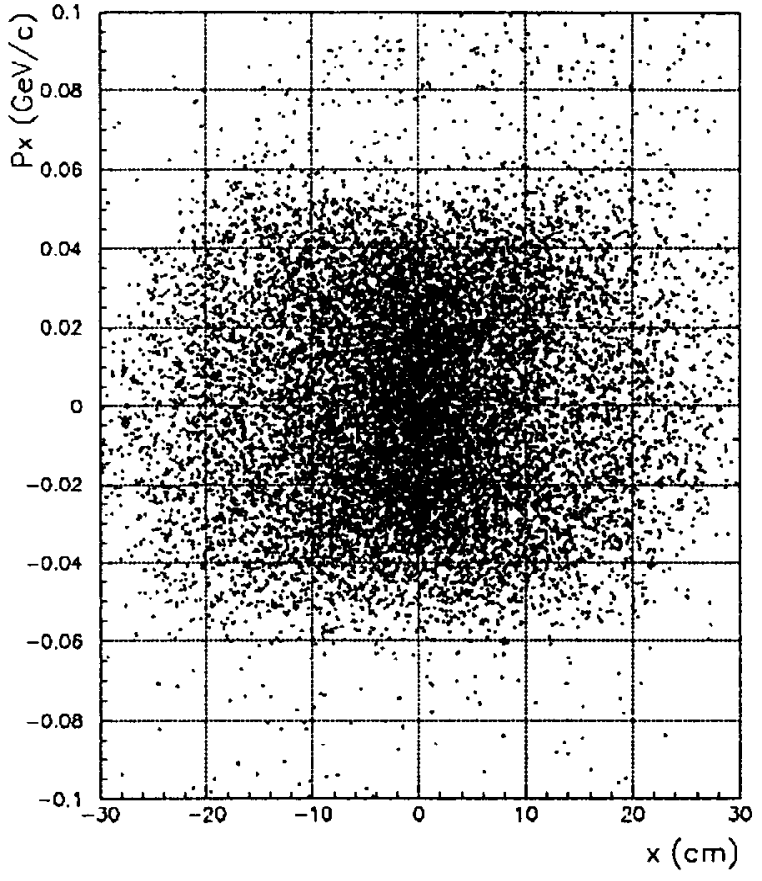

Figure 8: Horizontal phase space scatter plot for $\pi^{+}$at $\mathrm{z}=5 \mathrm{~m}$.

\section{REFERENCES}

[1] C. M. Ankenbrandt et al., "Status of Muon Collider Research and Development and Future Plans," Fermilab-Pub-98/179 (1998)

[2] N. V. Mokhov, "The MARS Code System User Guide, Version 13 (95)," LANL Report LA-UR-98-5716 (1998); nucl-th/9812038 v2 16 Dec 1998; http://www-ap.fnal.gov/MARS/ 